\title{
Sphere Detection Technique: An Optimum Detection Scheme for MIMO System
}

\author{
Prajapati Rajeev \\ Department of Computer and \\ Electronics Engineering \\ Kantipur Engineering College \\ Tribhuvan University \\ Kathmandu, Nepal
}

\author{
Adhikari Prabhat \\ Department of Electronics and \\ Computer Engineering \\ Institute of Engineering \\ Tribhuvan University \\ Kathmandu, Nepal
}

\author{
Lama Norsang \\ Department of Computer and \\ Electronics Engineering \\ Kantipur Engineering College \\ Tribhuvan University \\ Kathmandu, Nepal
}

\begin{abstract}
In this paper, various multiple input and multiple output (MIMO) detection techniques have been compared on the basis of BER performance and complexity. Maximum likelyhood (ML) detection method has shown optimal solution in MIMO systems compared to conventional detection techniques. However, higher receiver complexity leads to use of lower complexity techniques such as zero forcing ( $\mathrm{ZF}$ ) and minimum mean square error (MMSE) having relatively poor performance. Successive interference cancellation combined with ZF or MMSE has much improved performance but vulnerable to error propagation. Ordered succesive interference cancellation with MMSE (MMSE-OSIC) has reduced error propagation probability and gave the better performance. A new detection technique sphere detection (SD) based on Schnorr-Euchner enumeration has provided ML solution with much less computational complexity. For simulation, Rayleigh channel model has been considered.
\end{abstract}

\section{Keywords}

Multiple Input and Multiple Output (MIMO) System, Zero Forcing (ZF), Minimum Mean Square Error (MMSE), Successive Interference Cancellation (SIC), Maximum Likelihood (ML), Sphere Decoder

\section{INTRODUCTION}

Multiple Input Multiple Output (MIMO) is the use of multiple antennas at both the transmitter and receiver to improve communication performance. The terms input and output refer to the radio channel carrying the signal, not to the devices having antenna. MIMO technology has been shown to be one of the most promising emerging wireless technologies that can efficiently boost the data transmission rate, improve system coverage, and enhance link reliability. By employing multiple antennas at transmitter and receiver sides, MIMO system enable a new dimension - the spatial dimension - that can be utilized in different ways to combat the impairments of wireless channels.

As opposed to traditional wireless systems, in which there is one transmitting and one receiving antenna, MIMO systems use arrays of multiple antennas at both transmitter and receiver, all operating at the same frequency at the same time. This introduces spatial diversity into the system, which can be used to tackle the problem of multipath. In wireless communications system, such as point to point radio links, radio waves do not simply propagate from the transmit antenna to the receive antenna. Rather they bounce and scatter off objects, this effect is known as multipath. This effect is regarded as hindrance to the accurate transmission of data in traditional wireless links. MIMO systems exploit multipath by using the rich scattering environment to increase the spectral efficiency and/or reliability of the wireless system. There are different ways of exploiting multiple antennas at both ends of the MIMO communication link. For example, to achieve the best transmission reliability possible, the transmit antennas should be used such that transmit diversity is achieved. The transmission rate achieved in this case is normally comparable to that achieved in single-input singleoutput (SISO) systems. That is, all the degrees of freedom of the MIMO channel are used for improving the transmission reliability and not the transmission rate. An alternative is to use the transmit antennas to maximize the transmission rate. In this case, independent signals are transmitted from the different transmit antennas, i.e., there is no correlation among the transmitted signals from different antennas. While this approach increases the transmission rate, the corresponding reliability is poor. A combination of these two approaches is also possible, that is, one could trade rate for reliability or vice versa. The use of MIMO systems and associated signaling approaches are critical for the future of high data rate, extremely reliable wireless communications.

Maximum Likelihood is the optimum detection scheme considered here. It has high computational complexity hence time consuming for decoding. It has non-linear procedure and basically consists of a exhaustive search through all possible signal vectors. The complexity increases with increase in the number of antennas or the size of constellation. It is commonly known that at the receiver side the best performance can be achieved when a full maximum likelihood (ML) search is performed over the complete dimensions. It is obvious that the complexity of such a receiver grows exponentially with the size of the symbol constellation and/or antenna numbers. So, the receiver scheme with less complexity is needed without much performance loss. In general less complex receiver schemes result in a performance loss. The fundamental problem of MIMO systems is the mapping operation at the transmitter and the corresponding inversion at the receiver to optimize the overall performance of the wireless system.

Although the ML estimate is a very accurate means of estimating the transmitted symbols, it is clear that for higher order systems the computational complexity of ML would become very significant. The high computational complexity necessary for ML detector operation led to research into other detection algorithms which offer much lower complexity though they 
might be sub-optimal in nature. The basic idea of Sphere Decoder is to limit search only to the lattice points that lie in a sphere of radius around the given vector $y$ and in this way save on computations. It is clear that the closest point inside the sphere is also the closest point in the lattice. Therefore, there is no need to make an exhaustive search over all lattice points. Moreover, if the radius of sphere is properly chosen one can limit the number of operations used in order to find the desired point in sphere.

\section{METHODOLOGY 2.1 MIMO System Model}

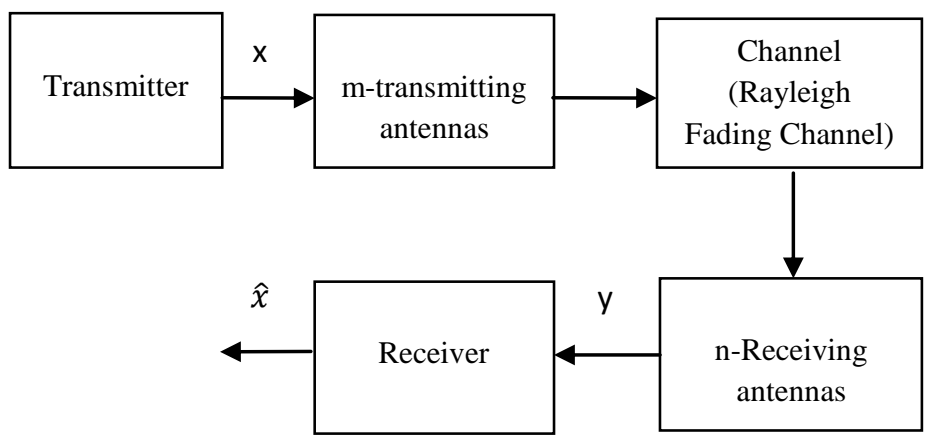

Fig 1: Overall System Block Diagram

The basic block diagram of Spatially Multiplexed MIMO (SM MIMO) system with $m$ transmit and $n$ receive antennas is shown in Figure 1. In a single user MIMO model with $m$ transmit and $n$ receive antennas, the MIMO system equation is given by,

$$
\left[\begin{array}{c}
\mathrm{y}_{1} \\
\mathrm{y}_{2} \\
\vdots \\
\vdots \\
\mathrm{y}_{\mathrm{n}}
\end{array}\right]=\left[\begin{array}{cccc}
\mathrm{h}_{11} & \mathrm{~h}_{12} & \ldots \ldots & \mathrm{h}_{1 \mathrm{~m}} \\
\mathrm{~h}_{21} & \mathrm{~h}_{22} & \ldots \ldots & \mathrm{h}_{2 \mathrm{~m}} \\
\vdots & \vdots & & \vdots \\
\mathrm{h}_{\mathrm{n} 1} & \vdots & \ldots \ldots & \vdots \\
\mathrm{h}_{\mathrm{n} 2} & \cdots \cdots & \mathrm{h}_{\mathrm{nm}}
\end{array}\right]\left[\begin{array}{c}
\mathrm{x}_{1} \\
\mathrm{x}_{2} \\
\vdots \\
\vdots \\
\mathrm{x}_{\mathrm{m}}
\end{array}\right]+\left[\begin{array}{c}
\mathrm{n}_{1} \\
\mathrm{n}_{2} \\
\vdots \\
\vdots \\
\mathrm{n}_{\mathrm{n}}
\end{array}\right] \ldots \ldots .(1)
$$

In the matrix form,

$$
\boldsymbol{y}=\boldsymbol{H} \boldsymbol{x}+\boldsymbol{n}
$$

Where $\boldsymbol{H}$ is the channel matrix of size $n \times m, \boldsymbol{y}$ is the $n \times 1$ received signal vector, $\boldsymbol{x}$ is the $m \times 1$ transmitted signal vector, and $\boldsymbol{n}$ is $n \times 1$ additive white Gaussian noise vector with zero mean and variance $\sigma^{2}$.

\subsection{Symbol Detection}

The symbol detection problem considered is the problem of estimating the MIMO channel input vector $\mathbf{x}$ given the received vector $\mathbf{y}$ assuming that the receiver has perfect knowledge of channel $(\mathrm{H})$. During transmission, the actual value of the transmitted signal constellation is shifted by noise. The aim of the receiver is to estimate as close a value to the transmitted symbol as possible. In essence, the receiver would need to decide on which value of the signal constellation is closest to the actual value of the transmitted signal. This decision is made on a symbol by symbol basis without taking into account any statistical dependencies that may be present in the sequence of vectors $\mathbf{x}$. In other words, we exclude coding across the time dimension and consider only the modulation-demodulation problem as depicted in Fig 1. The goal is to minimize the probability of decision error.

$$
P_{e}=P_{r}\{\hat{x} \neq x\}
$$

Where $\hat{x}$ is the demodulator's estimate of $\mathrm{x}$.

\subsubsection{Maximum Likelihood (ML) Receiver}

The output of ML receiver is given by

$$
\hat{x}=\arg \min _{x \in \mathcal{A}^{m}}\left\{\|y-H x\|^{2}\right\}
$$

Thus, the ML detector chooses the message which yields the smallest distance between the received vector $y$ and the hypothesized message. The search for the smallest distance for ML detection increases the complexity with increase in constellation size and/or number of transmits antennas. For QAM constellation alphabet with $\mathrm{M}$ transmit antennas, the receiver has to perform search over a set of size. So for higher-order modulation such as 16-QAM this complexity can become prohibitive for a small number of transmit antennas.

\subsubsection{Zero Forcing (ZF) Receiver}

Zero Forcing is linear detection scheme which forces the interference to zero. However it may result in increase in noise level. ZF output is given by

$$
\hat{x}=Q\left(W_{Z F} y\right)
$$

where, $\quad \mathrm{W}_{\mathrm{ZF}}=\left(\mathrm{H}^{\mathrm{H}} \mathrm{H}\right)^{-1} \mathrm{H}=\mathrm{H}^{+}$

$\mathrm{H}^{+}$denotes Moore-Penrose pseudo inverse of $\mathrm{H}$, which is generalized inverse that exists even when $\mathrm{H}$ is rank-deficient. The ZF receiver performs well in the high SNR regime, whereas in the low SNR regime there is significant noise enhancement.

\subsubsection{Minimum Mean Square Error (MMSE)}

Receiver

Unlike ZF, MMSE attempts to minimize both interference and noise at the same time. This results in better performance than ZF but with little additional complexity. The output of MMSE receiver is given by

$$
\hat{x}=Q\left(W_{M M S E} y\right)
$$

Where, $W_{M M S E}$ is chosen to minimize $E\left\{\left\|W_{M M S E} y-x\right\|^{2}\right\}$.

\subsubsection{ZF/MMSE-OSIC}

Three consecutive phases take place for SIC ordering ZF or MMSE receivers in [10] and [11]:

- Linear interference suppression

- Interference cancellation of the sub streams detected

- Reordering of the detection process through SNR postdetection

\subsubsection{Sphere Detection}

The sphere radius constraint can be included in the ML detection rule as,

$$
\hat{x}=\arg \min _{x \in \mathcal{A}^{m}}\left\{\|y-H x\|^{2} \leq R^{2}\right\}
$$

Where, $R$ is the radius of the sphere.

\subsection{Detection Algorithms}

The general algorithms for various detection algorithms are as follows:

1. Specify the number of transmit and receive antennas.

2. Specify the SNR value

3. Generate the transmit signal vector using appropriate constellation set (16QAM) 
4. Generate the channel matrix

5. Generate noise and compute the received signal

6. Specify one of the detection scheme and find the estimate of transmit signal vector

7. Compare the estimated signal with the transmit signal to find error.

8. Simulations are done for BER vs SNR on different detection schemes.

\section{SIMULATION AND RESULTS 3.1 Simulation Setup}

For the purpose of experimental setup, point to point (single user MIMO) is considered. The evaluation of performance of various detection algorithms is carried out under the variations of following scenarios:

- MIMO System: 2x2 and 4x4 MIMO

- Modulation: 16 QAM

- Channel: Rayleigh Fading Channel

- $\quad$ Noise: AWGN with zero mean and variance $\sigma^{2}$

\subsection{Simulation Results}

For the study of MIMO system, first the capacity of the system for various antenna systems i.e. Single Input Single Output (SISO), Single Input Multiple Output (SIMO), Multiple Input Single Output (MISO) and MIMO was performed. Also, comparison of BER vs SNR for different detection algorithms was performed.

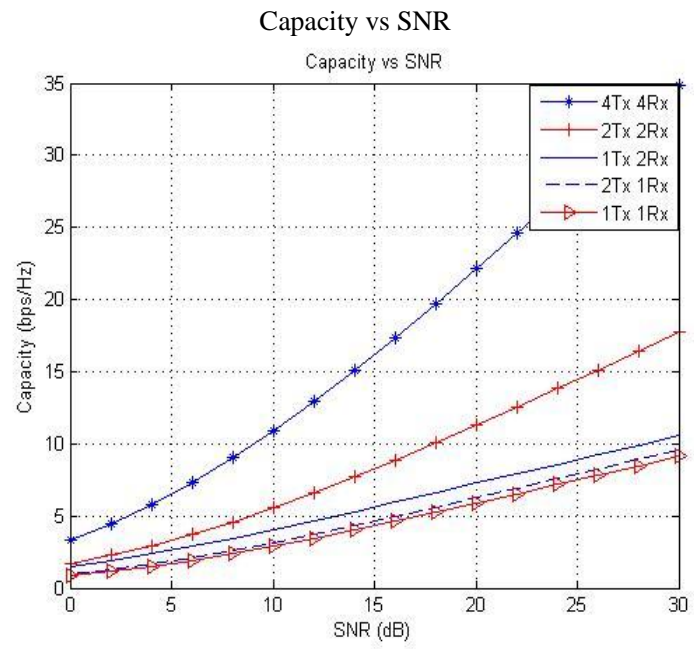

Fig 2: Comparison of capacity of SISO and MIMO system

Fig 2 shows the simulation result of comparison of capacity of various antenna configurations system. The channel capacity is least for single antenna, SISO system and increases with increase in the antenna numbers.
BER of ZF, ZF-SIC, ZF-OSIC for (4,4) MIMO system with 16-QAM modulation

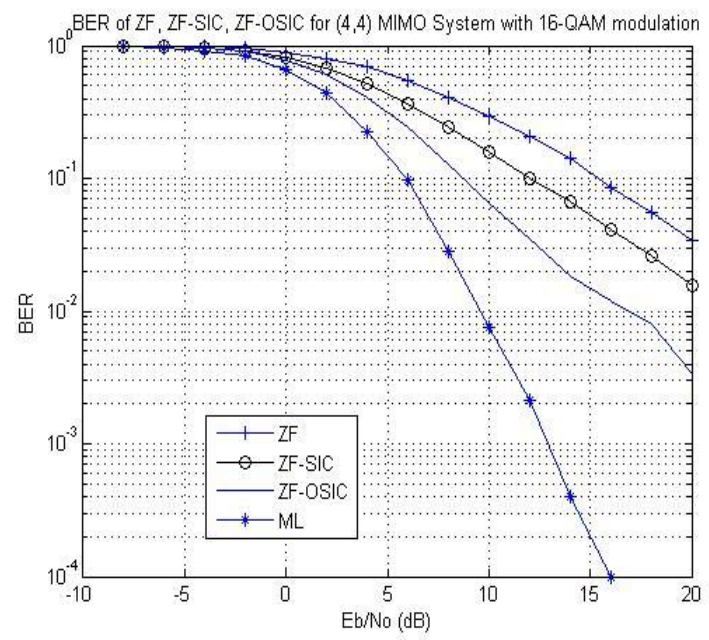

Fig 3: BER of ZF, ZF-SIC, ZF-OSIC for $(4,4)$ MIMO system with 16-QAM modulation

BER of MMSE, MMSE-SIC, MMSE-OSIC for $(4,4)$ MIMO system with 16QAM modulation

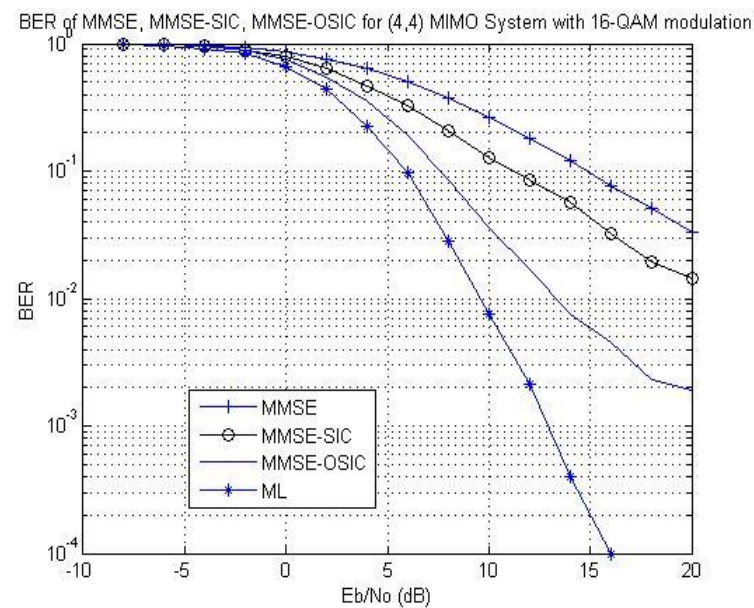

Fig 4: BER of MMSE, MMSE-SIC, MMSE-OSIC for $(4,4)$ MIMO system with 16-QAM modulation

BER of ZF-OSIC and MMSE-OSIC for $(4,4)$ MIMO system with 16-QAM modulation

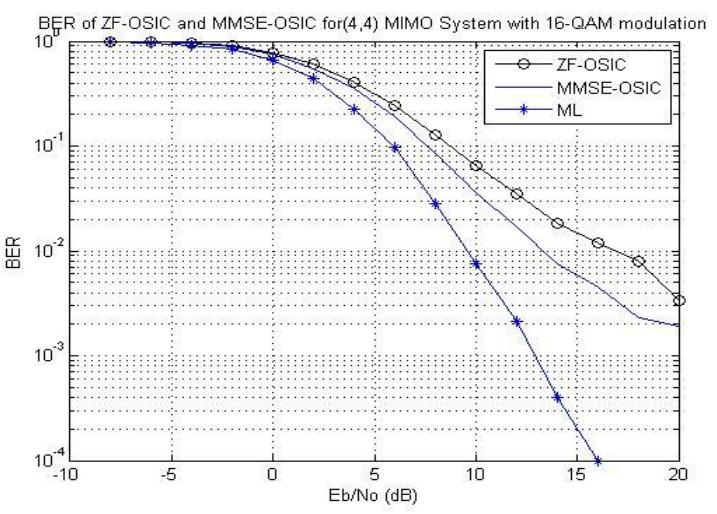

Fig 5: BER of ZF-OSIC and MMSE-OSIC for $(4,4)$ MIMO system with 16-QAM modulation 


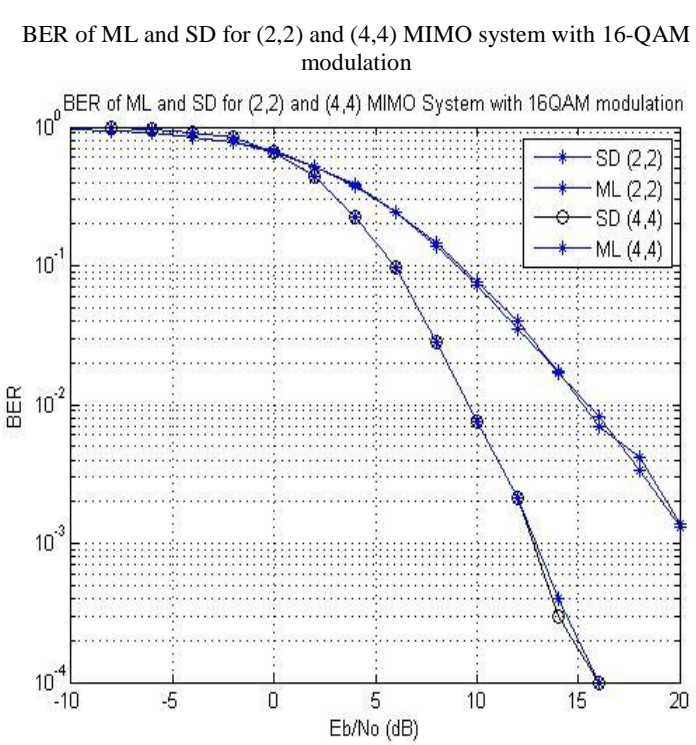

Fig 6: BER of ML and SD for $(2,2)$ and $(4,4)$ MIMO system with 16-QAM modulation

Fig 6 shows the performance curve of Sphere Decoder receivers with Schnorr Euchner enumeration in $(2,2)$ and $(4,4)$ MIMO system. It is seen that the output of SD is almost same as that of ML detection scheme. It would have been in exact with ML solution but since these schemes are run in different environment there is a slight deviation from the expected output.

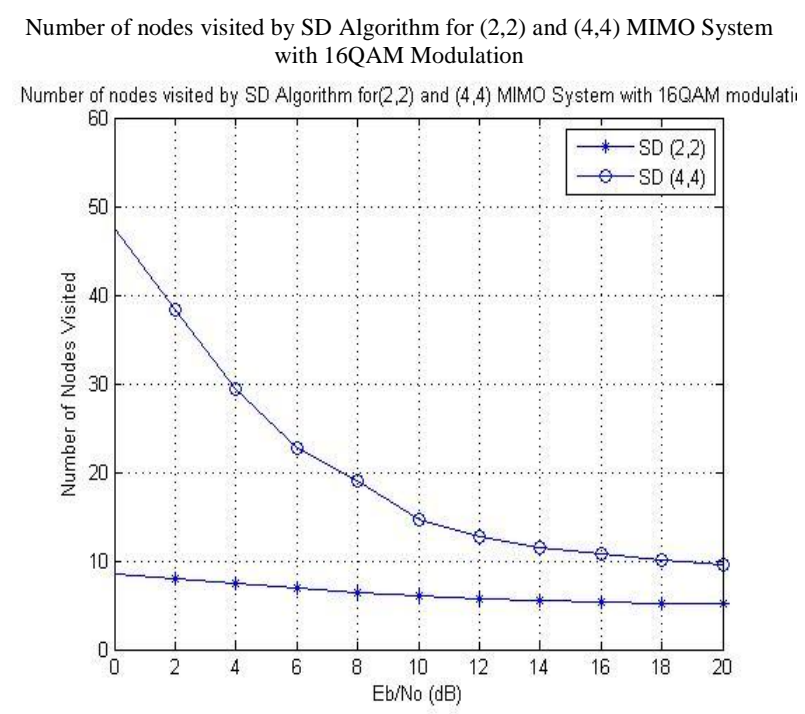

Fig 7: Number of nodes visited by SD algorithm for $(2,2)$ and $(4,4)$ MIMO system with 16-QAM modulation.

Fig 7 shows the average number of nodes visited by SD algorithm at various SNR values. It was found that the average number of visited nodes decreases as the SNR values increase. This was due to less probability of error in higher SNR regime and the decoder is likely to reach the solution more quickly.
Table 1: A comparitive table for different detection algorithms at $E_{b} / N_{0}=10 \mathrm{~dB}$ for 10000 iterations for $(4,4)$ MIMO System with 16 QAM modulation

\begin{tabular}{|c|c|c|c|}
\hline $\begin{array}{c}\text { Detection } \\
\text { Scheme }\end{array}$ & BER & $\begin{array}{c}\text { Runtime } \\
(\mathbf{s e c})\end{array}$ & $\begin{array}{c}\text { No. Of Nodes } \\
\text { Visited }\end{array}$ \\
\hline ZF & 0.2993 & 7.3172 & NA \\
\hline MMSE & 0,2616 & 7.5434 & NA \\
\hline ZF-SIC & 0.1577 & 10.3566 & NA \\
\hline MMSE-SIC & 0.1322 & 12.8834 & NA \\
\hline ZF-OSIC & 0.0623 & 12.8834 & NA \\
\hline MMSE-OSIC & 0.0366 & 13.4639 & NA \\
\hline SD & 0.0063 & 20.6215 & 14.94 \\
\hline ML & 0.0063 & 5062.2 & 65536 \\
\hline
\end{tabular}

Table 1 shows the BER performance and computational time of various detection techniques. The BER of SD is same as that of ML detection scheme with much less computational time as well as number of visited nodes.

\section{CONCLUSIONS}

In this paper different MIMO detection techniques was analysed and compared. The performance plots of linear receivers: ZF and MMSE were found relatively poor with slight improved performance of MMSE over ZF. In an attempt to improve the performance over linear receiver, successive interference cancellation with $\mathrm{ZF}$ and MMSE receiver was employed. SIC approach showed an improved performance but was vulnerable to error propagation. In an attempt to decrease the error propagation probability the ordered SIC was employed which increased the performance. It was found that MMSE-OSIC has the most favorable solution compared to previous detection methods.

Also, sphere decoder based on the Schnorr-Euchner enumeration was implemented to obtain maximum likelihood solution of the transmitted symbol at the receiver. This decoder along with other MIMO receiver was compared to conventional ML receiver by comparing their error performance. The sphere detection scheme was found to provide the ML solution with much less computational complexity.

\section{ACKNOWLEDGEMENT}

We would like to thank Department of Computer and Electronics Engineering, Kantipur Engineering College, Tribhuvan University and Department of Electronics and Computer Engineering, Pulchowk Campus, Tribhuvan University, Nepal for providing us with an opportunity to carry out this research work along with their valuable suggestions and guidance.

\section{REFERENCES}

[1] R. Prajapati, N. Lama, S. Bhandari, R.Rijal, "Adaptive Modulation in MIMO System", Proceedings of 7th International Conference on Software, Knowledge, 
Information Management and Applications (SKIMA2013), Chiang Mai University, Thailand, December 2013

[2] R. Gupta and A. Grover, "BER performance analysis of MIMO systems using equalization techniques," Innovative Systems Design and Engineering, vol. 3, no. 10,2012

[3] T. M. Duman and A. Ghrayeb, "Coding for MIMO communication systems," John Wiley and Sons Inc., 2007

[4] M. Rupp, G. Gristsch and H. Weinrichter, "Approximate ML detection for MIMO systems with very low complexity," Acoustics, Speech, and Signal Processing, 2004.Proceedings. (ICASSP '04). IEEE International Conference, Vol. 4, pp 809-812, 2004

[5] Y. H. Kim, B. Y. Cho and J.Y. Kim, "Wireless communication: trend andtechnical issues for MIMOOFDM system," Advanced Trends in Wireless Communications, 2011
[6] B. Holter, "On the capacity of the MIMO channel- A tutorial introduction," Norwegian University of Science and Technology, 2002

[7] L. G. Barbero and J. S. Thompson, "Performance of the Complex Sphere Decoder in Spatially Correlated MIMO Channels", Institute for Digital Communications. The University of Edinburgh, 2006

[8] Y. S. Cho, J. Kim, W.Y. Yang and C. G. Kang, "MIMOOFDM Wireless Communications with MATLAB," John Wiley and Sons Inc., 2010

[9] D. Wibben, R. Bohnke, V. Kuhn and K. D. Kammeyer, "Near-Maximum Likelihood detection of MIMO systems using MMSE-Based Lattice-Reduction," University of Bremen,2004

[10] G. Birlew, "Performance evaluation of a layered spacetime structure for MIMO systems," Addis Ababa University, July 2011

[11] G. Tsoulos, "MIMO system technology for wireless communications," CRC press, pp 105-108, 2006 